\title{
Clinical Heart Failure Patients with Ischemic Stroke Have a High Incidence $(>60 \%)$ of Atrial Fibrillation or Flutter Whether Systolic Function Is Preserved or Depressed
}

\author{
Jacob I. Haft*, Louis E. Teichholz \\ Department of Cardiology, Hackensack University Medical Center, Hackensack, USA \\ Email: *ihaft@aol.com
}

Received 24 June 2014; revised 26 July 2014; accepted 10 August 2014

Copyright (C) 2014 by authors and Scientific Research Publishing Inc.

This work is licensed under the Creative Commons Attribution International License (CC BY). http://creativecommons.org/licenses/by/4.0/

\section{(c) (i) Open Access}

\begin{abstract}
Clinical congestive heart failure (CHF) is a major risk factor for strokes. Patients with CHF commonly have atrial fibrillation or flutter (AF), which is frequently associated with, may be a marker for, and may be the mechanism of, ischemic strokes. To determine whether stroke patients with CHF have a high incidence of AF (that may be intermittent and not diagnosed), we reviewed all the 12 lead ECGs in a fourteen year institutional ECG data base and the clinical records and the available echocardiograms of 985 patients who had an ischemic stroke over a 3-year period. We found that $31.3 \%$ of the stroke patients had evidence of AF. Clinical congestive heart failure was present in 168 stroke patients; $61.9 \%$ of these stroke patients with CHF had evidence of AF. In the total stroke population, patients with other risk factors for stroke (hypertension, advanced age, diabetes, coronary artery disease) had an increased incidence of AF; but among stroke patients with CHF, only advanced age ( $\geq 75$ years) in addition to CHF increased the incidence of AF. To determine whether only the CHF stroke pts with systolic dysfunction had a high incidence of AF, we compared the incidence of AF in the $41.5 \%$ of the CHF patients with a depressed ejection fraction with the AF incidence in the $58.5 \%$ of CHF stroke patients with a normal ejection fraction $(\geq 50 \%)$. The incidence of $\mathrm{AF}$ was the same $(63.4 \%$ vs. $60.2 \%, \mathrm{p}=0.741)$ whether the ejection fraction was depressed or normal. These findings suggest that AF is common in patients with CHF who have strokes whether the ejection fraction is normal or depressed. CHF patients who have strokes and who are in sinus rhythm should be meticulously investigated for intermittent AF, so anticoagulation can be administered to prevent a further stroke.
\end{abstract}

\footnotetext{
"Corresponding author.
} 


\section{Keywords}

\section{Congestive Heart Failure, Ischemic Stroke, Atrial Fibrillation}

\section{Introduction}

Patients with heart failure frequently have strokes [1]-[3]; most strokes are considered ischemic [4]. The suggested mechanism for these strokes has included embolization of LV mural thrombi, spontaneous clots in the left atrium related to abnormalities of coagulation in patients (pts) with heart failure [5], periods of cerebral hypoperfusion due to low cardiac output and the usual causes of cerebral emboli including carotid disease, aortic endothelial ulcers and plaques, and thrombosis in the left atrial appendage in pts with atrial fibrillation. Atrial fibrillation is commonly found in pts with ischemic stroke and although it may be the cause for stroke, it might be just a marker for stroke risk. The physiologic abnormalities associated with heart failure also cause atrial fibrillation or flutter (AF), including elevation of the left ventricular end diastolic pressure, elevation of left atrial pressure, increase in left atrial wall stress, left atrial dilatation, enlargement, and left atrial fibrosis [6]. As heart failure is one of the major risk factors for stroke, it is also an important risk factor for AF [6]. AF is an accepted risk factor for stroke, especially if it is associated with hypertension, advanced age, heart failure, diabetes, vascular disease, or a prior stroke or TIA [7]. Pts with AF who have these risk factors are more prone to ischemic stroke and benefit from anticoagulation prophylaxis [8]. Intermittent AF increases the risk of stroke similarly to persistent or permanent AF [9] and intermittent AF that may have played a role in a stroke is frequently undiagnosed. It may be that AF is much more common in these ischemic stroke pts and that AF, frequently intermittent, may be the mechanism whereby the stroke risk factors, especially heart failure, cause stroke in many pts. It is important to diagnose that $\mathrm{AF}$ has been present in stroke pts because anticoagulation in these pts can significantly decrease the incidence of a recurrent stroke [10].

The aim of this study was to determine the incidence of AF in ischemic stroke pts who have clinical heart failure by reviewing all their 12 lead ECGs in a 14-year institutional data base in addition to their clinical history. We also determined if the presence of clinical heart failure with a depressed ejection fraction (systolic heart failure) or a preserved ejection fraction (also called "diastolic heart failure") affected the incidence of AF in these stroke pts.

This work has been partially presented at the 2012 Heart Failure meetings [11] and at other meetings [12] [13].

\section{Methods}

The clinical records of all patients discharged from Hackensack University Medical Center with a diagnosis of ischemic stroke (codes 434.00, 434.01, 434.11, 434.91) from January 1, 2005 to December 31, 2007 were reviewed. Patients with a mitral valve prosthesis or mitral stenosis were excluded. The diagnosis of clinical congestive heart failure (CHF) (codes 428.xx), hypertension (codes 401-5.xx), diabetes (codes 249.xx), and coronary artery disease (CAD) (history of myocardial infarction (codes 410.xx), angioplasty (code 45.82), coronary artery bypass grafting (code 45.81) and age and sex were tabulated. All of the 12 lead ECGs in the institutional data base from 1993 through July 2007 for each patient were printed out and reviewed (1 to 56 ECGs per patient, approximately 6000 ECGs reviewed) and the presence of atrial fibrillation (irregularly irregular ventricular rhythm in the absence of $\mathrm{P}$ waves with or without irregularly irregular undulation of the baseline) or flutter (regular rapid flutter waves with fixed ratio or irregular ventricular depolarizations) recorded on at least one ECG in the data base taken during the admission for stroke or on any emergency room visit, clinic visit or another admission before or after the admission for stroke, was determined. Thus we included pts with intermittent AF, many who had no clinical history of AF, in addition to those with persistent AF. The available echocardiogram reports were reviewed and the presence of left atrial enlargement (LAE) (dimension $\geq 4.0 \mathrm{~cm}$ ), left ventricular (LV) enlargement ( $L V$ end diastolic dimension $\geq 5.6 \mathrm{~cm}$ ), and abnormal ejection fraction $(<50 \%)$ were tabulated.

Statistics: The association between two groups of categorical variables were reviewed by $2 \mathrm{X} 2$ contingency tables and analyzed by Fishers exact test with two sided $\mathrm{p}$ values $<0.05$ considered statistically significant; $95 \%$ confidence limits of individual proportions were determined (modified Wald method) (Graph Pad Software Inc.) 
The incidence of atrial fibrillation or flutter (AF) in these ischemic stroke patients with and without one or more of the variables: age $>75$, gender, CHF, hypertension, coronary artery disease and diabetes, left atrial enlargement, left ventricular enlargement, ejection fraction $<50 \%$ were compared and the Odds Ratio (O.R.) (19932013 MedCalc Software bvba) was determined. The effect of the other risk factors on the incidence of AF in patients with CHF was determined by comparing the AF incidence in the CHF patients with and without each of the risk factors. The ischemic stroke patients with clinical CHF were divided into those with heart failure with depressed ejection fraction $(<50 \%)$ (HFDEF), and those with heart failure with preserved ejection fraction, ( $\geq 50 \%$ ) (HFPEF) and the incidence of AF was compared between these groups. The effect of the other risk factors on the incidence of AF was determined in the HFDEF and HFPEF groups and compared between groups.

\section{Results}

\subsection{Total Ischemic Stroke Population}

Ischemic stroke was diagnosed in 985 patients discharged from January 1, 2005 to December 31, 2007. AF was found in 306 (31.3\%). Heart failure was present in 168 patients (17.1\%); (61.9\% had AF), 784 patients had hypertension (33.7\% with AF), 527 patients were 75 years old or older (45.2\% AF), 240 patients had CAD (42.9\% $\mathrm{AF})$, and 204 patients had diabetes (39.2\% AF). In the total population of patients with ischemic stroke, the patients with any of the risk factors for stroke (also the risk factors for AF) had a significantly increased incidence of AF compared to those who did not have the risk factor ( $<0.0001$ to $\mathrm{p}=007)$. The incidence of AF was highest in the ischemic stroke patients with CHF $(61.9 \%, \mathrm{p}<0.0001))$.

There were 937 patients with available echocardiographic diagnoses. Left atrial enlargement (LAE) was present in 527 patients, $56.2 \%$ of the stroke pts. Patients with LAE had a $47.3 \%$ incidence of AF, significantly greater than $11.7 \% \mathrm{AF}$ among those without LAE $(\mathrm{p}<0.0001)$. Among those with ejection fraction $(\mathrm{EF})$ less than $50 \%, 49.0 \%$ had AF, compared to $28.3 \%$ with $\mathrm{EF} \geq 50 \%$ ( $<<0.0001)$. Thus in the total stroke group, patients with left atrial enlargement or with depressed ejection fraction had a significantly higher incidence of AF compared to those without these echo findings. The incidence of AF was not significantly higher in those with LV enlargement (E) compared to those without LVE.

\subsection{Heart Failure Population (Table 1, Figure 1)}

Among the 168 stroke patients with heart failure, except for age $\geq 75$ years, the effect of adding each of the other clinical variables, hypertension, coronary artery disease or diabetes, did not significantly increase the incidence of AF. In stroke patients with heart failure who were 75 years or older, AF occurred in $67.7 \%$ compared to $42.1 \%$ in patients with CHF younger than $75(\mathrm{p}=0.0073)$.

Patients with each of the clinical risk factors who also had CHF had a significantly higher incidence of AF than patients with the same risk factor that did not have CHF.

Echocardiographic diagnoses were available in 159 of the 168 patients with clinical CHF. Patients with clinical heart failure who had left atrial enlargement had a significantly higher incidence of AF than those with a normal size left atrium $(70.3 \%$ vs $25.8 \%, p=0.0002)$. In the total population of stroke patients with CHF, the presence or absence of LV enlargement did not affect the incidence of AF (65.5\% vs 62.3\%, p $=0.8335$ ).

Thus the presence of clinical congestive heart failure in a patient with ischemic stroke carries with it the highest incidence of AF with only the presence of advanced age adding to the likelihood of finding AF in stroke patients with CHF. Heart failure patients with hypertension, with CAD or with diabetes had a similar incidence of AF regardless of whether hypertension, CAD, or DM were present or absent. The presence of LA enlargement increased the incidence of AF but the presence of LV enlargement in the stroke pts with CHF did not.

\subsection{Ejection Fraction and HFDEF Compared to HFPEF (Table 2, Table 3, Figure 2)}

Of the 159 patients with clinical heart failure who had echo reports available, 66 patients had EF < 50\% (HFDEF) and 93 patients had a normal ejection fraction (HFPEF). Thus in the stroke patients with CHF significantly more patients had HFPEF than HFDEF (58.5\% vs $41.5 \%$, p < 0.0001). Comparing stroke patients with clinical heart failure who had decreased ejection fraction with those with preserved ejection fraction, more of the patients with HFPEF were women (69.8\% vs $36.6 \%, \mathrm{p}<0.0002)$. More of the HFDEF patients had CAD and had LV enlargement. There was a tendency toward more patients with LA enlargement in the HFDEF group 
Table 1. Influence of other clinical risk factors on AF incidence in stroke Pts with CHF.

\begin{tabular}{|c|c|c|c|c|c|}
\hline \multirow{2}{*}{ Group } & \multicolumn{3}{|c|}{ Patients } & \multirow{2}{*}{$\mathrm{p}=$} & \multirow{2}{*}{ Comment } \\
\hline & $\mathrm{AF}$ & Total & $\mathrm{AF} \%$ & & \\
\hline $\mathrm{CHF} \geq 75$ у.о. & 88 & 130 & $67.69 \%$ & 0.0073 & (95\% CL 59.23, 75.14\%, O.R. 2.88) \\
\hline $\mathrm{CHF}<75$ y.o. & 16 & 38 & $42.11 \%$ & & \\
\hline $\mathrm{CHF}+\mathrm{Hi} \mathrm{BP}$ & 93 & 152 & $61.18 \%$ & 0.7875 & \\
\hline CHF Nl BP & 9 & 16 & $56.25 \%$ & & \\
\hline $\mathrm{CHF}+\mathrm{CAD}$ & 41 & 72 & $56.94 \%$ & 0.2654 & \\
\hline CHF no CAD & 63 & 96 & $65.63 \%$ & & \\
\hline $\mathrm{CHF}+\mathrm{DM}$ & 27 & 51 & $52.94 \%$ & 0.1233 & \\
\hline CHF no DM & 77 & 117 & $65.81 \%$ & & \\
\hline $\mathrm{CHF}+\mathrm{LAE}$ & 90 & 128 & $70.31 \%$ & $<0.0001$ & (CL 61.88\%, 77.56\%, O.R. 6.81) \\
\hline CHF no LAE & 8 & 31 & $25.81 \%$ & & \\
\hline $\mathrm{CHF}+\mathrm{LVE}$ & 19 & 29 & $65.52 \%$ & 0.8335 & \\
\hline CHF no LVE & 81 & 130 & $62.31 \%$ & & \\
\hline$\geq 75+\mathrm{CHF}$ & 88 & 130 & $67.69 \%$ & $<0.0001$ & (CL 59.23, 75.14\%, O.R. 3.45) \\
\hline$\geq 75$ no $\mathrm{CHF}$ & 150 & 397 & $37.78 \%$ & & \\
\hline $\mathrm{Hi} \mathrm{BP}+\mathrm{CHF}$ & 95 & 152 & $62.50 \%$ & $<0.0001$ & (CL 54.58, 69.81\%, O.R. 4.57) \\
\hline Hi BP no CHF & 169 & 632 & $26.74 \%$ & & \\
\hline $\mathrm{CAD}+\mathrm{CHF}$ & 41 & 72 & $56.94 \%$ & 0.0055 & (CL 45.44, 67.75\%, O.R. 2.22) \\
\hline CAD no CHF & 62 & 168 & $36.90 \%$ & & \\
\hline $\mathrm{DM}+\mathrm{CHF}$ & 27 & 51 & $52.94 \%$ & 0.0307 & (CL 39.52, 65.95\%, O.R. 2.12) \\
\hline DM no CHF & 53 & 153 & $34.64 \%$ & & \\
\hline
\end{tabular}

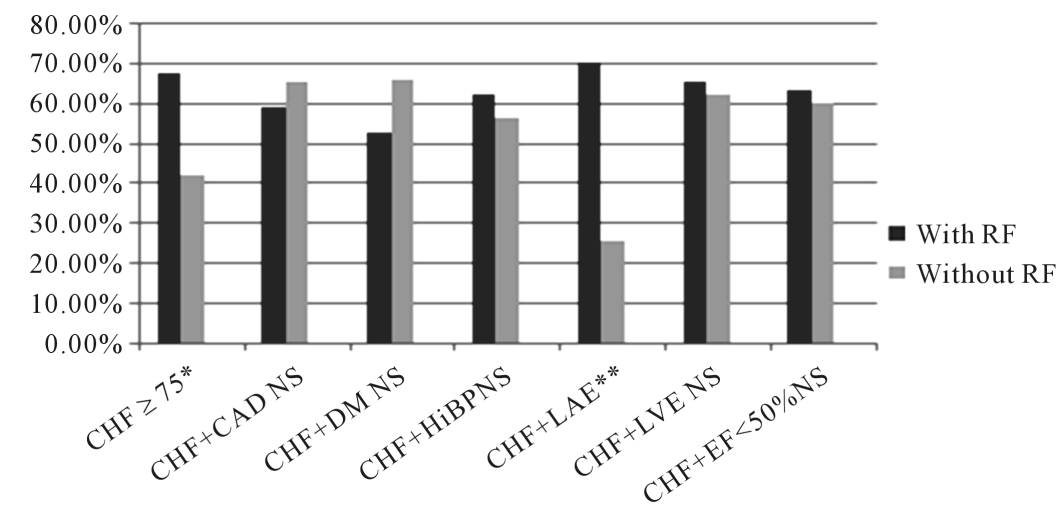

Figure 1. Stroke patients with CHF: Incidence of AF in patients with CHF and with each of the other risk factors. Except for age and left atrial enlargement the presence or absence of the other risk factors in addition to clinical CHF had no significant influence on the incidence of AF. Abbreviations: AF-atrial fibrillation or flutter; $\mathrm{CAD}$ - coronary artery disease; $\mathrm{CHF}$ — clinical congestive heart failure; DM - diabetes; EF — ejection fraction; LA - left atrium; LAE-left atrial enlargement; LV-left ventricle; LVE-left ventricular enlargement; NS-not significant; Pts-patients; RF-risk factor. ${ }^{*} \mathrm{p}<0.01,{ }^{* *} \mathrm{p}<0.0001$. 
Table 2. Incidence of clinical stroke risk factors in stroke patients with CHF with regard to ejection fraction $<50 \%$.

\begin{tabular}{ccccccccc}
\hline \multirow{2}{*}{ Group } & \multicolumn{3}{c}{ Patients } & \multicolumn{3}{c}{ Patients } & O.R. & $\mathrm{p}=$ \\
\cline { 2 - 6 } & HFDEF & Total & HFDEF\% & HFPEF & Total & HFPEF\% & & \\
\hline Total Patients & 66 & 159 & $41.51 \%$ & 93 & 159 & $58.49 \%$ & 1.98 & 0.0035 \\
Women & 24 & 66 & $36.36 \%$ & 62 & 93 & $66.67 \%$ & 3.50 & 0.0002 \\
$\geq 75$ & 50 & 66 & $75.76 \%$ & 73 & 93 & $78.49 \%$ & & 0.7042 \\
Hi BP & 63 & 66 & $95.45 \%$ & 84 & 93 & $90.32 \%$ & & 0.3617 \\
CAD & 39 & 66 & $59.09 \%$ & 36 & 93 & $38.71 \%$ & 2.29 & 0.0154 \\
DM & 18 & 66 & $27.27 \%$ & 31 & 93 & $33.33 \%$ & & 0.4862 \\
LAE & 58 & 66 & $87.88 \%$ & 70 & 93 & $75.27 \%$ & 2.38 & 0.0667 \\
LVE & 27 & 66 & $40.91 \%$ & 2 & 93 & $2.15 \%$ & 31.50 & $<.0001$ \\
\hline
\end{tabular}

Table 3. Incidence of AF in stroke patients with CHF with regard to ejection fraction, effect of risk factors.

\begin{tabular}{|c|c|c|c|c|c|c|c|}
\hline \multirow{2}{*}{ Group } & \multicolumn{3}{|c|}{ Patients } & \multicolumn{3}{|c|}{ Patients } & \multirow{2}{*}{$\mathrm{p}=$} \\
\hline & HFDEF & $\mathrm{AF}$ & $\mathrm{AF} \%$ & HFPEF & $\mathrm{AF}$ & $\mathrm{AF} \%$ & \\
\hline Total Patients & 66 & 42 & $63.64 \%$ & 93 & 56 & $60.22 \%$ & 0.7414 \\
\hline Women & 24 & 16 & $66.67 \%$ & 62 & 37 & $59.68 \%$ & 0.6264 \\
\hline Men & 42 & 22 & $52.38 \%$ & 31 & 19 & $61.29 \%$ & 0.4831 \\
\hline$\geq 75$ years old & 50 & 36 & $72.00 \%$ & 73 & 47 & $64.38 \%$ & 0.4347 \\
\hline$<75$ years old & 16 & 6 & $37.50 \%$ & 20 & 9 & $45.00 \%$ & 0.7412 \\
\hline Hi BP & 63 & 41 & $65.08 \%$ & 84 & 51 & $60.71 \%$ & 0.6098 \\
\hline nlBP & 3 & 1 & $33.33 \%$ & 9 & 5 & $55.56 \%$ & 1.0000 \\
\hline CAD & 39 & 25 & $64.10 \%$ & 36 & 18 & $50.00 \%$ & 0.2491 \\
\hline No CAD & 27 & 17 & $62.96 \%$ & 57 & 38 & $66.67 \%$ & 0.8079 \\
\hline DM & 18 & 11 & $61.11 \%$ & 31 & 15 & $48.39 \%$ & 0.5536 \\
\hline No DM & 48 & 31 & $64.58 \%$ & 62 & 41 & $66.13 \%$ & 1.0000 \\
\hline LAE & 58 & 41 & $70.69 \%$ & 70 & 49 & $70.00 \%$ & 1.0000 \\
\hline No LAE & 8 & 1 & $12.50 \%$ & 23 & 7 & $30.43 \%$ & 0.6417 \\
\hline LVE & 27 & 19 & $70.37 \%$ & 2 & 0 & $0.00 \%$ & \\
\hline No LVE & 39 & 23 & $58.97 \%$ & 91 & 56 & $61.54 \%$ & 0.8456 \\
\hline
\end{tabular}

(87.9\% vs 75.3\%, $\mathrm{p}=0.0667$ ). The incidence of hypertension, diabetes and age $\geq 75$ years was not different between the groups.

In the stroke patients with clinical CHF the incidence of AF was the same whether EF was normal or less than $50 \%(60.2 \%$ vs $63.36 \%, p=0.7414)$. This similarity of incidence of AF in stroke patients with clinical heart failure who had HFDEF or HFPEF was present regardless of gender, or whether hypertension, CAD, diabetes or age $\geq 75$ were present. Although there was a trend toward LAE in more patients with HFDEF than HFPEF the incidence of AF was the same in the patients with LAE whether the patients had HFDEF or HFPEF. Although in the general stroke population patients with decreased ejection fraction had a higher incidence of AF, in patients with clinical congestive heart failure there was no difference in the incidence of AF in the HFDEF patients compared to the HFPEF patients. This may be because patients with clinical CHF have increased LA stress and 


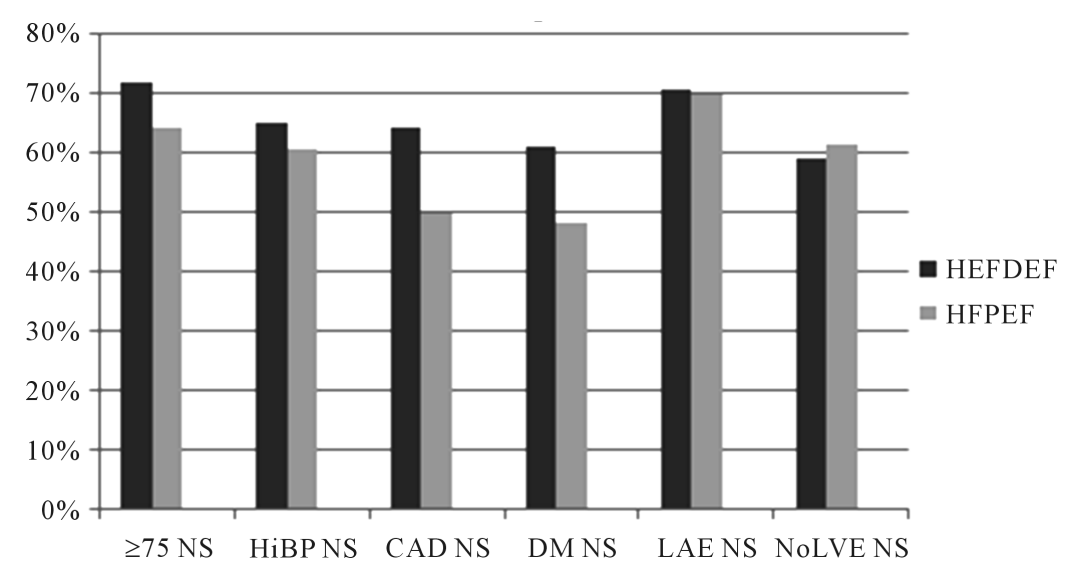

Figure 2. Stroke patients with CHF: Incidence of AF in CHF stroke patients with depressed ejection fraction or preserved ejection fraction. There was no significant difference in the incidence of AF between the pts with clinical heart failure with a depressed ejection fraction $(<50 \%)$ or with a preserved ejection fraction regardless of the presence of any of the stroke risk factors. Abbreviations as on previous figures and HFDEF-heart failure with depressed ejection fraction, HFPEF-heart failure with preserved ejection fraction.

LA pressure due to elevated LV end-diastolic pressure regardless of the ejection fraction. In the total stroke population clinical CHF was diagnosed in a higher percentage of patients with depressed EF (66 of 153 patients, 43.15\%) whereas CHF was diagnosed in only 93 of the 784 patients with normal EF $(11.86 \%)$ p $<0.0001$.

Thus, heart failure with preserved ejection fraction was more common in pts with clinical CHF. The incidence of AF was the same in those with HFPEF compared to patients with depressed ejection fraction and remained the same in the presence or absence of any of the stroke risk factors or of left atrial enlargement. Ejection fraction, whether preserved or depressed does not appear to affect the increased propensity to AF in these stroke patients with clinical CHF.

\section{Discussion}

Since the report of the Framingham study in 1978 [14], nonvalvular AF has been implicated as a risk factor for strokes. Many studies [15] have confirmed this relationship and have demonstrated that anticoagulation will significantly decrease the incidence of ischemic stroke in patients with AF. Prospective studies in patients with AF have identified risk factors associated with AF that increase the likelihood of stroke and that are associated with an increase in the prophylactic benefit of anticoagulation [15]. These studies have led to the development of various schema for determining which patients with non-valvular AF will benefit from anticoagulation [16]. A major problem in determining the actual importance of AF in stroke, however, has been the fact that intermittent AF carries the same risk of stroke as persistent or permanent AF [9] [10] [17]. This has raised the possibility that occult intermittent AF that is not present on admission to the hospital in many stroke patients may be playing a major role in the occurrence of stroke and that AF is underdiagnosed [18]. Although the occurrence of AF may be just a marker for a higher propensity for stroke (the presence of AF around the time of stroke in pts with intermittent AF has not been consistently demonstrated) AF might be the mechanism for stroke and is certainly a marker for the use of warfarin to prevent stroke. Transient AF with stasis in parts of the atrium might lead to the formation of a clot that embolises and causes a stroke some time later after sinus rhythm has been spontaneously restored. Alternatively, a clot might have embolised during a transient period of AF but the rhythm reverted to sinus before the patient arrived in the hospital and an ECG had been recorded. Identification of those stroke patients in whom AF may have played a role is important because patients with AF who have had a stroke are at high risk for a recurrent stroke (greater than $10 \%$ per year [19], and will benefit from anticoagulation [20].

There have been many reports of patients monitored after strokes to determine if intermittent AF is occurring [18] [21]-[23]. The longer the period of monitoring after a stroke, the higher is the incidence of the diagnosis of transient intermittent AF. The highest incidence of transient AF in post stroke patients has been found in stroke patients with implanted pacemakers or defibrillators that have an intrinsic Holter monitoring capability [24] [25]. 
Ziegler et al. [24] prospectively followed cryptogenic stroke patients with implanted devices for $1.1+/-0.7$ years and found intermittent AF to occur in $28 \%$ of these patients who had no diagnosable cause for their stroke. They did not comment on the importance of AF in their total population of stroke patients, but if the patients excluded from their analysis because they had documented AF, and those excluded because they were on warfarin or because they were on antiarrhythmic drugs (presumably because they may have had AF) are added to those of the cryptogenic stroke group who had AF it appears that over $63.0 \%$ of patients with stroke had definite or probable AF as a marker or as the mechanism for their stroke. We retrospectively studied 128 ischemic stroke patients who had implanted permanent pacemakers or defibrillators using their charted clinical diagnosis of AF and the findings in the ECG data base and found that $71.1 \%$ had evidence of AF [20] [25]. Of those with the clinical diagnosis of AF 86.8\% also had ECGs in the data base that confirmed the clinical diagnosis of AF largely obviating the possibility that AF had been diagnosed because of artifact on the Holter-type recording of the device. We also found patients who had ECGs showing AF who did not have the clinical diagnosis of AF [25], suggesting it was missed on reading the monitor record from the device. The high incidence of AF found in the ECG database may have been because patients with pacemakers or with defibrillators were not normal; they also interface with the medical center frequently and have ECGs performed more often. The findings in these studies suggest that AF, very often intermittent, may play a much more important role in ischemic stroke and may be a marker for stroke or may be the common mechanism whereby the stroke risk factors cause stroke. Although the incidence of stroke in patients with AF may be 10\%/year or less, depending on the presence of risk factors, the incidence of AF in patients with ischemic stroke may be as high as $>60 \%$.

In this study, using discharge diagnoses and a 14-year ECG data base, we have found that $31.3 \%$ of the patients who present with an ischemic stroke have evidence of AF. This is greater than the $15 \%-19 \%$ that has been considered the incidence of AF in stroke patients [26], and is probably because AF may be intermittent and may not be present at the time of the stroke admission to the hospital. This $31.3 \%$ incidence probably is an underestimation of the actual incidence of AF since the diagnosis of AF was based largely on the serendipitous recording of an ECG during an episode of AF that occurred during another interface with the hospital [27]. Among patients with each of the risk factors for stroke, hypertension, advanced age, diabetes, coronary artery disease, and congestive heart failure, the incidence of AF was significantly higher than in the stroke patients without the risk factors.

Of the risk factors for ischemic stroke, CHF was associated with the highest incidence of AF $(61.9 \%$, 95\% confidence limits $54.4 \%, 68.9 \%$ ). This may be related to the pathophysiological abnormalities associated with clinical heart failure (an elevation of left atrial pressure with increased wall stress, atrial dilatation and fibrosis due to an elevation of left ventricular end-diastolic pressure) that also can cause AF. Alternatively or in addition, patients with clinical heart failure tend to present to the hospital more often and with more ECGs recorded, diagnosing intermittent AF is more likely. In the stroke patients with CHF the presence or absence of hypertension, diabetes or coronary disease did not significantly affect the incidence of AF. In the stroke patients with clinical CHF, only age 75 years old or older significantly increased the likelihood of finding AF (67.7\%). Echocardiographically, the heart failure pts with LA enlargement had a higher incidence of AF than those with a normal LA (70.3\%, odds ratio $=6.81, \mathrm{p}<0.0001)$.

Over the past few years, it has been demonstrated that at least $40 \%$ of patients with clinical CHF do not have left ventricular systolic dysfunction. The CHF pts with normal systolic function have the same prognosis as those with CHF due to systolic dysfunction. We found that the incidence of AF in stroke pts with clinical CHF was the same whether failure was due to systolic dysfunction or not. (Pts with clinical CHF with normal systolic function have been diagnosed as having "diastolic heart failure" and various criteria for the diagnosis of diastolic failure have been studied and presented. The diagnostic criterion that is most universally accepted is "heart failure with a preserved ejection fraction” which we have used (HFPEF)).

In the stroke patients with clinical CHF significantly more patients had HFPEF than HFDEF (58.5\% vs 41.5\%, $\mathrm{p}<0.0001)$ and more of the patients with HEFPEF were women. The incidence of AF was the same in the HFPEF group compared to the HFDEF patients $(60.2 \%$ vs $63.4 \%, \mathrm{p}=0.7414)$ and remained the same whether there was associated hypertension, coronary artery disease or diabetes. In the patients $\geq 75$ years old with HFDEF the incidence of AF was higher than among those who were younger, but in the HFPEF group age did not significantly alter the incidence of AF in these stroke pts. The incidence of LAE tended to be higher in the HFDEF patients ( $87.9 \%$ vs $75.3 \%$, p $=0.0667)$ but the incidence of AF was the same in both the HFPEF and HFDEF patients with LAE, and though significantly lower in the patients without LAE the incidence of AF was 
also not significantly different in the HFDEF or HFPEF patients with normal size left atria. The incidence of LAE in the patients with AF in the HFDEF group was not statistically significantly different from those with AF in the HFPEF group $(97.6 \%$ vs $87.5 \%, p=0.0758$.) Thus HFPEF is more common than HFDEF in patients with ischemic stroke. The incidence of AF is the same in stroke patients with HFPEF and HFDEF who have clinical heart failure. Since patients with clinical heart failure have increased LA stress and a high incidence of LAE it is not unexpected that the incidence of AF in these patients would be similar regardless of the LV ejection fraction.

The WARCEF study [28] can be interpreted to support our findings of a high incidence of AF in patients with CHF who have ischemic strokes. The study prospectively compared the use of warfarin or aspirin in 2305 patients with systolic heart failure (ejection fraction $\leq 35 \%$ ) who were in sinus rhythm, and who were followed for $3.5+/-1.8$ years. Although the composite endpoint of death, ischemic stroke, or intracerebral or intracranial hemorrhage was not significantly different in the two groups, the incidence of ischemic stroke was significantly less in the warfarin treated patients $(2.5 \%$ vs $4.7 \% \mathrm{p}=0.005)$. Using their numbers: based on the stroke incidence in the group not receiving warfarin, if warfarin had been withheld from the warfarin group 54 ischemic strokes would have been expected to occur $(55 \times 1142 / 1163=54), 25$ more than the 29 reported to have a stroke; i.e. 25 strokes had been prevented by being in the warfarin group. However the patients on warfarin had INRs $\geq 2$ only $72.9 \%$ of the time. Thus if all of the warfarin-treated patients had always been adequately anticoagulated 34 strokes would have been prevented $(25 / 0.729=34)$. If we assume that warfarin mainly prevents strokes in patients with atrial fibrillation, AF would have been implicated as the cause for stroke in 34 of the 54 patients who would have been expected to have a stroke or $63.0 \%$, similar to the incidence of AF we found in our CHF pts. In their discussion the authors note that the observed relative reduction in ischemic stroke to be "similar to that observed among patients with atrial fibrillation” [28].

The possible limitations of our study include that it was retrospective using discharge diagnoses. It was conducted in a single center, and the ECGs in the data base were performed randomly, with no set schedule. The finding of AF was fortuitous and serendipitous and possibly underestimated the incidence of intermittent AF.

\section{Conclusions}

Using a 14-year ECG data base, we have found that ischemic stroke patients have a high incidence of AF that may be frequently intermittent and not diagnosed. The incidence of AF varies with the presence of risk factors and is highest $(61.9 \%)$ in patients with clinical heart failure. AF may be a marker for the propensity for stroke but may be the common mechanism whereby the stroke risk factors cause stroke.

The majority of stroke patients who have CHF with or without systolic dysfunction (decreased ejection fraction) have evidence of AF. The occurrence of AF in CHF stroke patients was especially high in patients who are 75 or older or who have left atrial enlargement. Since stroke patients with AF are at great risk for recurrent stroke, it is important to meticulously investigate stroke patients with CHF in whom AF has not been diagnosed, so anticoagulation can be considered to prevent a recurrent stroke if AF is found. Moreover, patients with CHF who complain of palpitations should be studied to diagnose possible AF so anticoagulation can be considered to prevent a first stroke.

\section{References}

[1] Appelros, P. (2006) Heart Failure and Stroke. Stroke, 37, 1637. http://dx.doi.org/10.1161/01.STR.0000227197.16951.2b

[2] Witt, B.J., Brown, R.D., Jacobsen, S.J., Weston, S.A., Ballman, K.V., Meverden, R.A. and Roger, V.L. (2006) Ischemic Stroke after Heart Failure: A Community-Based Study. American Heart Journal, 152, 102-109. http://dx.doi.org/10.1016/j.ahj.2005.10.018

[3] Alberts, V.P., Bos, M.J., Koudstaal, P.J., Hofman, A., Witteman, J.C.M., Stricker, B.H.C. and Breteler, M.M.B. (2010) Heart Failure and the Risk of Stroke: The Rotterdam Study. European Journal of Epidemiology, 25, 807-812. http://dx.doi.org/10.1007/s10654-010-9520-y

[4] Roger, V.L., Go, A.S., Lloyd-Jones, D.M., Benjamin, E.J., Berry, J.D., Borden, W.B., et al. on Behalf of AHA Statistics Committee and Stroke Statistics Subcommittee (2012) Heart Disease and Stroke Statistics—2012 Update: A Report from the American Heart Association. Circulation, 125, e2-e220. http://dx.doi.org/10.1161/CIR.0b013e31823ac046

[5] Lip, G.Y.H. and Gibbs, C.R. (1999) Does Heart Failure Confer a HypercoagulableState? Virchows Triad Revisited. Journal of the American College of Cardiology, 33, 1424-1426. 
[6] Fuster, V., Ryden, L.E., Cannom, D.S., Crijns, H.J., Curtis, A.B., Ellenbogen, K.A., et al. (2006) ACC/AHA/ESC 2006 Guidelines for the Management of Patients with Atrial Fibrillation: A Report of the American College of Cardiology/American Heart Association Task Force on Practice Guidelines and European Society of Cardiology Committee for Practice Guidelines (Writing Committee to Revise the 2001 Guidelines for the Management of Patients with Atrial Fibrillation): Developed in Collaboration with the European Heart Rhythm Association and the Heart Rhythm Society. Circulation, 114, e257-e354. http://dx.doi.org/10.1161/CIRCULATIONAHA.106.177292

[7] Stroke Risk in Atrial Fibrillation Working Group (2008) Comparison of 12 Risk Stratification Schemes to Predict Stroke in Patients with Nonvalvular Atrial Fibrillation. Stroke, 39, 1901-1910. http://dx.doi.org/10.1161/STROKEAHA.107.501825

[8] Hart, R.G.G., Benavente, O., McBride, R. and Pearce, L.A. (1999) Antithrombotic Therapy to Prevent Stroke in Patients with Atrial Fibrillation: A Meta-Analysis. Annals of Internal Medicine, 131, 492-501. http://dx.doi.org/10.7326/0003-4819-131-7-199910050-00003

[9] Hohnloser, S.H., Pajitnev, D., Pogue, J., Healey, J.S., Pfeffer, M.A., Yusuf, S., et al. (2007) Incidence of Stroke in Paroxysmal versus Sustained Atrial Fibrillation in Pts taking Oral Anticoagulation or Combined Antiplatelet Therapy: An Active W Substudy. Journal of the American College of Cardiology, 50, 2156-2161.

[10] Singer, D.E., Albers, G.W., Dalen, J.E., Go, A.S., Halperin, J.L., Manning, W.J., et al. (2008) Antithrombotic Therapy in Atrial Fibrillation: American College of Chest Physicians Evidence Based Practice Guidelines (8th Edition). Chest, 133, 546S-592S.

[11] Haft, J.I. and Teichholz, L.E. (2012) Atrial Fibrillation/Flutter Is Found in over 60\% of Heart Failure Patients Who Have Strokes. Journal of Cardiac Failure, 18, S97.

[12] Haft, J.I. and Teichholz, L.E. (2012) Atrial Fibrillation or Flutter Is the Common Mechanism Whereby the Stroke Risk Factors Cause Stroke. Chest, 142, A110a.

[13] Haft, J.I. and Teichholz, L.E. (2012) Atrial Fibrillation or Flutter Is Found in 45.4\% of Elderly Patients with Nonhemorrhagic Stroke. Stroke, 43, A2349.

[14] Wolf, P.A., Dawber, T.R., Thomas, H.E. and Kannel, W.B. (1978) Epidemiologic Assessment of Chronic Atrial Fibrillation and Risk of Stroke: The Framingham Study. Neurology, 28, 973-977. http://dx.doi.org/10.1212/WNL.28.10.973

[15] Pisters, R., Lane, D.A., Marin, F., Camm, A.J. and Lip, G.Y.H. (2012) Stroke and Thromboembolism in Atrial Fibrillation-Systematic Review of Stroke Risk Factors and Risk Stratification Schema. Circulation Journal, 76, 2789-2304.

[16] Lip, G.Y.H. (2012) Can We Predict Sstroke in Atrial Fibrillation? Clinical Cardiology, 35, S21-S27. http://dx.doi.org/10.1002/clc.20969

[17] Hart, R.G., Pearce, L.A., Rothbart, R.M., McAnulty, J.H., Asinger, R.W. and Halperin, J.L. (2000) Stroke with Intermittent Atrial Fibrillation: Incidence and Predictors during Aspirin Therapy. Journal of the American College of Cardiology, 35, 183-187. http://dx.doi.org/10.1016/S0735-1097(99)00489-1

[18] Liao, J., Zahira, K., Scallan, C., Morillo, C. and O’Donnell, M. (2007) Noninvasive Cardiac Monitoring for Detecting Paroxysmal Atrial Fibrillation or Flutter after Acute Ischemic Stroke. A Systematic Review. Stroke, 38, 2935-2940. http://dx.doi.org/10.1161/STROKEAHA.106.478685

[19] Gage, B.F., van Walraven, C., Pearce, L., Hart, R.G., Koudstaal, P.J., Boode, B.S.P. and Petersen, P. (2004) Selecting Patients with Atrial Fibrillation for Anticoagulation. Stroke Risk Stratification in Patients Taking Aspirin. Circulation, 110, 2287-2292. http://dx.doi.org/10.1161/01.CIR.0000145172.55640.93

[20] Haft, J.I. (2013) Stroke Prevention in Atrial Fibrillation: Impact of Novel Anticoagulants. Clinical and Applied Thrombosis/Hemostasis, 19, 241-248.

[21] Tayal, A.H., Tian, M., Kelly, K.M., Jones, S.C., Wright, D.G., Singh, D., Jarouse, J., Brillman, J., Murali, S. and Gupta, R. (2008) Atrial Fibrillation Detected by Mobile Cardiac Outpatient Telemetry in Cryptogenic TIA or Stroke. Neurology, 71, 1696-1701. http://dx.doi.org/10.1212/01.wnl.0000325059.86313.31

[22] Elijovich, L., Josephson, S.A., Fung, G.L. and Smith, W.S. (2009) Intermittent Atrial Fibrillation May Account for a Large Proportion of Otherwise Cryptogenic Strokes: A Study of 30-Day Cardiac Event Monitors. Journal of Stroke \& Cerebrovascular Diseases, 18, 185-189. http://dx.doi.org/10.1016/j.jstrokecerebrovasdis.2008.09.005

[23] Gladstone, D.J., Blakeley, J., Dorian, P., Fang, J., Silver, F.L. and Kapral, M.K. (2008) Detecting Paroxysmal Atrial Fibrillation after Ischemic Stroke and Transient Ischemic Attack: If You Don’t Look, You Won’t Find. Stroke, 39, e78e79. http://dx.doi.org/10.1161/STROKEAHA.107.513002

[24] Ziegler, P.D., Glotzer, T.V., Daoud, E.G., Wyse, D.G., Esekowitz, M.D., Koehler, J.L. and Hilker, C.E. (2010) Incidence of Newly Detected Atrial Arrhythmia via Implantable Devices in Patients with a History of Thromboembolic Events. Stroke, 41, 256-260. http://dx.doi.org/10.1161/STROKEAHA.109.571455

[25] Haft, J.I. (2012) The Importance of Atrial Fibrillation/Flutter as a Cause of Ischemic Stroke. International Journal of 
Cardiology, 158, 143-144. http://dx.doi.org/10.1016/j.ijcard.2012.04.036

[26] Wolf, P.A., Abbott, R.D. and Kannel, W.B. (1991) Atrial Fibrillation as an Independent Risk Factor for Stroke: The Framingham Study. Stroke, 22, 983-988. http://dx.doi.org/10.1161/01.STR.22.8.983

[27] Haft, J.I. and Teichholz, L.E. (2008) Echocardiographic and Clinical Risk Factors for Atrial Fibrillation in Hypertensive Patients with Ischemic Stroke. American Journal of Cardiology, 102, 1348-1351. http://dx.doi.org/10.1016/j.amjcard.2008.07.009

[28] Homma, S., Thompson, J.L., Pullicino, P.M., Levin, B., Freudenberger, R.S., Teerlink, J.R., Ammon, S.E., Graham, S., et al. (2012) Warfarin and Aspirin in Patients with Heart Failure and Sinus Rhythm. The New England Journal of Medicine, 366, 1859-1869. http://dx.doi.org/10.1056/NEJMoa1202299

\section{Abbreviations used in the Tables}

AF-atrial fibrillation or flutter;

CAD_ coronary artery disease;

$\mathrm{CHF}$ — clinical congestive heart failure;

cm-centimeter;

DM-diabetes mellitus;

Ej. Fraction-ejection fraction;

HiBP-hypertension;

HFDEF-heart failure with depressed ejection fraction $(<50 \%)$;

HFPEF - heart failure with preserved ejection fraction $(\geq 50 \%)$;

LAE-left atrial enlargement;

$\mathrm{L}$ atrium-left atrium;

LV-left ventricle;

LVE_-left ventricular enlargement;

No-the characteristic following is not present;

O.R.—odds ratio;

pts-patients;

y.o.- - years old. 
Scientific Research Publishing (SCIRP) is one of the largest Open Access journal publishers. It is currently publishing more than 200 open access, online, peer-reviewed journals covering a wide range of academic disciplines. SCIRP serves the worldwide academic communities and contributes to the progress and application of science with its publication.

Other selected journals from SCIRP are listed as below. Submit your manuscript to us via either submit@scirp.org or Online Submission Portal.
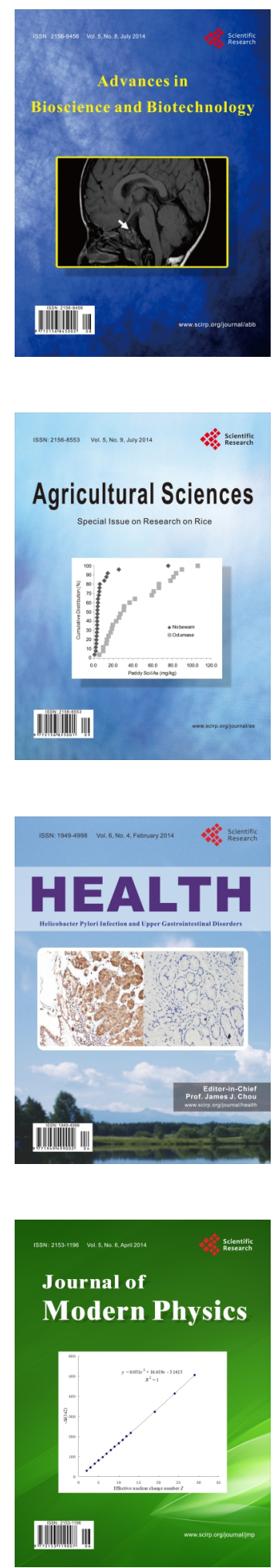
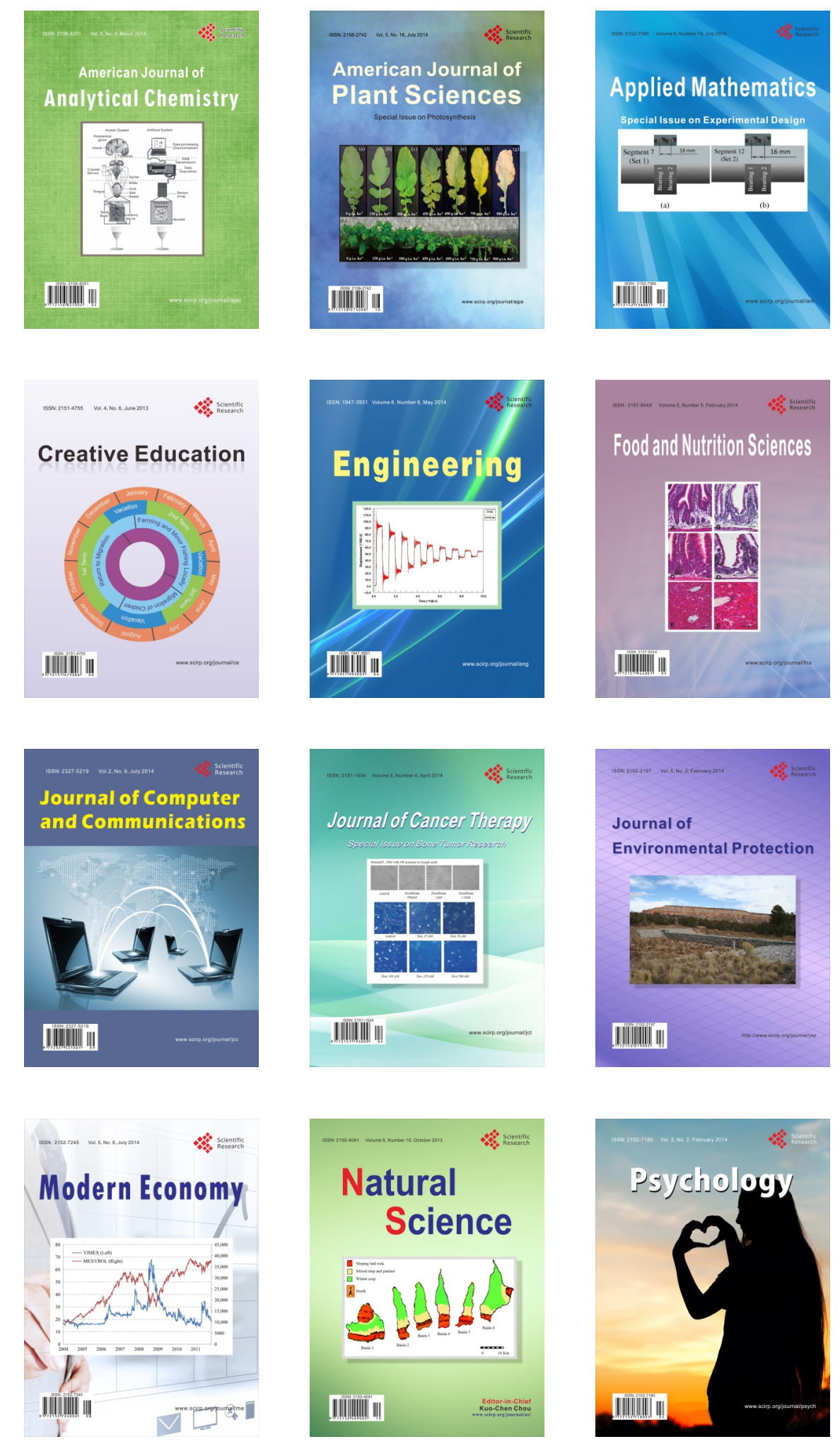